\title{
New technique for herniotomy in children-clinical trial
}

\author{
Omid Amanollahi ${ }^{1, ~ *, ~ D i a n a ~ N o e m i ~ D i a z ~}{ }^{2}$, Vahid Moetamedi ${ }^{3}$ \\ ${ }^{1}$ Department of Pediatric surgery, Mohammad Kermanshahi Hospital, Kermanshah University of Medical Sciences, Kermanshah, Iran \\ ${ }^{2}$ Madani Hospital, Zanjan University of Medical Sciences, Zanjan, Iran \\ ${ }^{3}$ Department of General Surgery, Imam Reza Hospital, Kermanshah University of Medical Sciences, Kermanshah, Iran
}

\section{Email address:}

oamanollahi@yahoo.com (O. Amanollahi), dianariel2001@yahoo.com (D. N. Diaz), Dr.v.moetamedi@kums.ac.ir (V. Moetamedi)

\section{To cite this article:}

Omid Amanollahi, Diana Noemi Diaz, Vahid Moetamedi. New Technique for Herniotomy in Children-Clinical Trial. Advances in Surgical Sciences. Vol. 2, No. 1, 2014, pp. 1-4. doi: 10.11648/j.ass.20140201.11

\begin{abstract}
Objective: to assessment of hernial sac ligation necessity after its transection in children herniotomy and compare the recurrence and complication rates between two methods of hernial repair in children (the classic method with ligation of the hernial sac and a new method without sac ligation). study design: this is a randomized controlled trial (RCT) Method: A total of 200 children ranging from neonates to 12 years of age, with inguinal hernia were admitted to Emam-Reza Hospital, Kermanshah, Iran between march 2011 and march2012, for elective hernial surgery The patients who were more prone to recurrence; such as patients with incarcerated hernia and increased intra-abdominal pressure were excluded from the study. After exclusion of some patients, 182children included in the study. They were randomly allocated into two groups, a study group (herniotomy without sac ligation) and a control group (herniotomy with sac ligation). Both groups were followed up for a period of 1-1.5 years postoperatively for recurrence or other complications. Results: There was only one case of recurrent hernia in the control group, and none in the study group. There was no statistically significant difference in the rate of recurrence or other complications between the two groups. Conclusions: This study showed that hernial sac ligation after its transection is not necessary in pediatric herniotomy. Omitting ligation did not result in any significant effect on early and late complication.
\end{abstract}

Keywords: Children, Herniotomy, Non Ligation, Hernial Sac, Recurrence

\section{Introduction}

Inguinal hernia is a common cause of patient referral for surgery. It's a common problem that seen in 2-3 percent of all fullterm neonates and even more in prematures. Its etiology in children is due to failure of closure of the processus vaginalis. The processus vaginalis is an embryonic developmental outpouching of the peritoneum. It is present from around the 3 th month of gestation, and

In boys, it leads the testis in their descent into the scrotom and then closes. It may happen before birth, or a few weeks after birth. Its portion that is around the testes named the tunica vaginalis

Failure of closure of the processus vaginalis leads to a number of abnormalities. Like inguinal hernia-hydrocele-cyst of spermatic cord. If the processus vaginalis doesn't close, there is the potential for an indirect inguinal hernia to develop, although not all cases with a patent processus vaginalis will develop hernia. But it increases the patient risk to develop a hernia. Persistent patent processus vaginalis is more common on the right than the left. .If it does not close in females, it forms the canal of Nuck

An inguinal hernia will not resolve by itself and all cases need surgery. Most hernias in children are indirect These hernias need to be repaired, because they are likely to become incarcerated.

Prompt surgery is needed for neonates and infants because risk of incarceration in these children is more than older one and adult.

Hernias that are incarcerated, even after reduction need to be repaired as soon as possible because of the risk of strangulation.

Surgery may be delayed on premature babies with hernias that are not incarcerated, because Premature neonates are at risk of anesthesia.

Traditional technic of surgery for inguinal hernia in children is hernialsac dissection, transection and high ligation with non absorbable or absorbable sutures, (classic treatment).(1)When the internal ring is very large,(for example when surgeon fingertip easily entere to internal ring, its aproximation and tightening of internal ring is 
recommended by most surgeons also.(2). Although cutting of the sac,that is pathway of fluid and vicera into inguinal canal, is mandatory and logical, but the need of its ligation is doubtful.

A hernia sac is continiuity of peritoneum into inguinal canal(process vaginalis) and it is a thin layer of pritoneum that easily adhere and fuses by itself after cutting. It seems that etiology of inguinal hernia in children is more depend on patency and continiuity of this pathway(hernial sac) and only cutting of this continiuity and its disturbing is enough to resolve problem. It's proved that all defects and laceration and transections of pritoneum closes itself, spontaneously and rapidly without any suture (3-4) . hence, there should not be any difference between the open peritoneum in herniotomy and other abdominal surgeries in which the peritoneum is left open and not closed separately .today its not emphesized on repairing pritoneal layer separately in references books of surgery. It looks like that high ligation of the sac is not an essential step in children inguinal hernial repair. Besides, performing an extra step may predispose to complications, and more time and cost spending. .Here we assess the effect of non-ligation method on recurrence rate during pediatric herniotomy.

\section{Materials and Methods}

This prospective study was conducted between march 2011 and 2012 in pediatric surgery ward of areferral hospital. A total of 200 children who admitted for elective inguinal herniotomy ( between neonacy to 12 years old age), enrolled andrandomly allocated into two group, (100 in each group) .study group (herniotomy without sacligation) and control group (herniotomy with sac ligation), totally 218 herniotomy was done in 200children(137right side,45 left side and18 cases of bilateral hernia) mean age in control group was 23 month and in study group 21 month, whit no meaning full difference between two groups. The ratio of boys to girl in control group was 6.1 to 1 and in study group 4.8 to 1 (no meaningful difference). After the baseline investigations, and after explanation of two method, informed consent of the parents was taken all cases of emergent surgery due to incarceration, and all patients who were more prone to recurrence ( increased intra-abdominal pressure, pritoneal dialysis, ventriculo pritoneal shunts, ascitis, soft tissue disorders,,,,) excluded from the study. In the study group (non ligation of hernial sac), the hernial sac was dissected and separated from thespermatic cord and simply transected and excised at the internal ring level, without ligation. afterexcision, proximal end of hernial sac retracted into pritoneal cavity without closing it by suture. Then skin sutured only and operation terminated. but in the control group (ligation of hernial sac) the hernial sac after its dissection and separation from spermatic cord was ligated and transfixed by nonabsorbable suture (silk3-0)material and excised at the internal ring level(classic method).in both groups, in few cases, if internal ring was large and patent (,Based on the surgeon's judgment, for example with entering the finger tip into the internal ring)it aproximated with one or two absorbable(vicryl3-0) suture, in our study it was 5 cases in control group and 4 cases in study group(in one case we have evisceration of intestine during non ligation method that managed only with returning of intestine to abdomen and aproximation of internal ring with two sutures , and patient has no recurrence in 1.5 years follow up.) all operations were done under general anesthesia by the same pediatric surgeon. No prophilactic or post op antibiotic were given to patients. Both group evaluated and compared during first few days after surgery for early complications, ( hematoma, bleeding, pain, wound infection, and spermatic cord inflammation, and after discharge all patients visited periodically at the hospital outpatient clinic for up to 1-1.5 years and assessed for signs and symptoms of hernial recurrence. If a patient didn't come for visit .we called them by telephone and invited them to visit in clinic and we could follow up all of them except two case who moved to another town and excluded from study. Criteria for diagnosis of recurrence was based on anamnestic information, parents observation and history, physical exam, and inguinal sonography. All collected data after coding entered to Excel sheet, using descriptive statistics, (average, standard deviation, confidence interval,) chi-square test and t-test were used to comparing results. The softwar used was, spss, version 18. This study has permission from ethical committe of university of medical sciences.

\section{Results}

One recurrent inguinal hernia was detected in controll group only during the 1.5 year follow-up period. There was only a case of bleeding in controll group who underwent re exploration in operation room and hemostasis (bleeding had subcutaneous origin)some cases had edema and swelling and stiffness of spermatic cord and, or testis. This condition is due to exessive manipulation of spermatic cord and probably thrombosis of spermatic veins and may increase incidence of ischemic orchitis and finally to testicular atrophie. The number of this complication in two group hadn't any meaningful difference. One of another complications after inguinal hernial surgery is re-accumulation of fluid in tunica vaginalis that's named post-operative hydrocele. This condition can be due to persistance of hernial sac continiuity and entrance of pritoneal cavity fluid into scrotom and tunica vaginalis (thechnical error in diagnosis and cutting the hernial sac) or it can be only a reactional fluid accumulation after surgery the first condition needs reoperation but last one usually respond to conservative management and one or two aspiration. This complication was also near same in two group and no meaningful difference founded in two group. wound infection isn't common in inguinal hernial surgery because inguinal herniotomy is a clean surgery, in all of our patients we had only5 cases of wound infection, totally in both group, without any meaningful differences between two groups. Almost all of them were superficial infaction 
who treated only with partial opening of skin (incisional site)and another complications had not meaningful differences in two groups (table1).all complications managed conservatively and medically except for reoperations for one recurrent hernia and 2 case of post operative hydroceles that persist after aspiration. We had not any mortality and no case of testicular atrophie.

Table 1. Compare of complications in both groups study group (non ligation of hernia sac), control group (ligation of hernia sac)

\begin{tabular}{lccc}
\hline Complication type & study group & control group & P-value \\
\hline Recurrence & 0 & 1 & 0.48 \\
Hemorage and Hematoma & 0 & 1 & 0.48 \\
Swelling of spermatic cord & 7 & 8 & 0.79 \\
Post operative hydrocele & 11 & 9 & 0.81 \\
Wound infection & 2 & 3 & 0.67 \\
\hline
\end{tabular}

\section{Discussion}

Although classic and standard treatment for inguinal hernia in children is excision and suture-ligation of the indirect sac but Our study demonstrated that non ligation of hernial sac has no effect on the recurrence rate. We proved that sac ligation is not absolutely necessary. hernial sac after transection closes rapidly by metamorphosis of the in-situ mesodermal cells as a part of peritoneum(5), thus there should not be any need to its ligation. Eliminating the passage of viscera into the inguinal canal and cut off the continuity of sac seems to be enough for indirect inguinal hernia tretment. in other hand, adhision of sac remnant suture in front of internal ring makes a fix point between their and prevent its complete retraction into abdominal cavity, hence increase possibility of recurrence.

although some reports emphesize on excision and high ligation of the indirect inguinal hernia sac as an essential part of the repair, , supposing that the omission of this step is insufficient treatment and will increase complications and recurrence, an analysis of 584 operations for recurrent inguinal hernia was made in an attempt to determine the cause of the recurrence based on the anatomic findings. The recurrence was indirect in 300 , direct in 241 , and various other in 43 operations. The causes of the indirect recurrences appeared to be an unrecognized hernia, incomplete dissection or improper ligation of the sac, failure to narrow the cord, or inadequate reconstruction of the internal ring. No cause for the diffuse direct recurrences was apparent. (6) but it seems over emphasized as an essential part of the repair and it has not been proven yet .Numerous clinical trials have confirmed that it is unnecessary to close the hernial sac that is a part of pritoneum with Sutures .. Shulman et al. demonstrated Invagination and excision of the hernia sac do not have adverse effects on repair integrity. They limit the dissection and reduce the morbidity and risk of injury to the spermatic cord and surrounded structures. They are safer and more appropriate for repair of sliding hernia. Ligation of the hernia sac in inguinal hernia surgery is not only unnecessary and time consuming but also leads to increased postoperative pain. Recurrence rates are statistically unaffected by not ligating the sacthe absence of adverse effects on herniorraphy without sac ligation in adults (7). A prospective study of laparoscopic inguinal hernia repair in children by Schier showed that there is no difference in suturing or not after the peritoneum was incised and the sac resected(8). Veena Kumari et al showed that closure of the hernial sac is not necessary in herniotomy associated with orchiopexy. Fifty cases of undescended testis, age ranging from eight months to 12 years were enrolled. All of them underwent standard orchiopexy without ligation of the hernial sac. Follow up of all cases ranged between 1.5 years to three years. Not a single case was reported with evidence of hernia. They concluded that, It is unnecessary to ligate the hernial sac during orchiopexy. (9). Delikoukos and Smedberg had been shown that non ligation of the indirect hernia sac in adults does not increase the chance of recurrence and patient has less pain in this method. Between January 1999 and December 2006, 477 patients with indirect inguinal hernia entered the study and were randomized to have high hernia sac ligation and excision (group $\mathrm{A}, \mathrm{n}=238$ ) or not (group $\mathrm{B}, \mathrm{n}=239$ ). The two groups were comparable regarding demographic data. Postoperative pain was associated with statistically significantly more episodes in group $1,27 \%(65 / 238)$, than in group 2, 10\% (24/239),

From the results of this study, it appears that we are able to demonstrate a significant benefit from the omission of high hernia sac ligation and excision on postoperative pain in patients who undergo tension-free indirect inguinal hernia mesh repair.(10-11)

Gharaibeh showed also that nonligation of hernial sac in adult herniorrhaphy does not increase recurrence, During the period from July 1987 to July 1990, a total of 60 consecutive patients with 64 indirect inguinal hernia were entered into this prospective study. The indirect inguinal hernia was repaired using Maloney technique with excision of the hernial sac proximally as deep as possible without any attempt to ligate or transfix the sac. All patients were followed up at regular intervals.

During the 3-year period, a total of 64 indirect inguinal hernia in 60 consecutive patients were repaired. Their ages ranged between 15-85 years (mean of 50 years). The follow up ranged from 9-12 years (a mean of 10 years and 5 months). Four patients died of unrelated causes. There was no clinically documented case of recurrence in the remaining 56 patients.

This study, although not controlled, confirmed that non-ligation of the proximal sac during indirect hernia repair was not associated with an increase rate of recurrence..(12)

We think that, non-ligation may be advantageous. It is more rapid with less dissection. It makes the repair more safe because prevents inadvertent ligation of spermatic cord elements and entrapement of viscera between sutures specially in sliding hernia. Nonligation may reduce infection and adhision rate because absence of suture material as a 
forigne body.CM, St Peter SD, in their research have cared for six children who have presented with latent inguinal or pelvic abscesses following high ligation of the inguinal hernia sac with silk suture. The purpose of their report was to examine the varied presentations by which latent complications following herniorraphy may manifest. They concluded that Utilization of braided, non-absorbable silk suture may result in latent abscess formation and the need for operative drainage of inguinal or pelvic abscess..(13-14), Our experience with pediatric herniotomy without ligation of the sac has been encouraging. This is the largest series presented in children herniotomy that includes younger children and girls, but further studies with more cases and longer follow-up should clarify the issue with or without sac ligation. This should lead to the establishment of evidence-based clinical guidelines of how the operation should be performed.

\section{References}

[1] Thomas R.Weber, Thomas F.Tracy jr, Marfin S. Keller, Groin hernia and hydrocele in: Keith W. Ashcraft, George Whitfield Holcomb, J Patrick Murphy .Editors. Pediatric Surgery 4th edition 2005

[2] Eric w.Fonkalsrud md. Disorders of inguinal canal in:James A O’Neill,jr,, Jay 1. Grosfeld, Eric W. Fonkalsrud, Arnold G. Coran, Anthony A. Caldamone, editors. Principles of Pediatric Surgery, second edition 2004 Missouri.mosby,inc. 1995: p.440.

[3] Ellis H., Heddle R. Does the peritoneum need to be closed at laparotomy? Br J Surg 1977.

[4] F. R. Johnson, H. W. Whitting, Repair of parietal peritoneum, British Journal of Surgery pages 653-660, May 1962Volume
49 , Issue 218

[5] Mohta A, Jain N,Iniraya KP, Saluja SS, Sharma S, Gupta A .Non ligation of the hernial sac during herniotomy: a prospective study. Pediatr surg int 2003

[6] Postlethwait RW. Recurrent inguinal hernia. Ann Surg 1985; 202: 777-779

[7] Shulman AG, Amid PK, Lichtenstein IL. Ligation of the hernia sac. A needless step in adult hernia. Pediatric surg int 2007; hernioplasty . Int surg 78:152-153.

[8] Aranda, Mario Riquelme-Q. Laparoscopic pediatric inguinal hernia repair: no ligation, just resection. Journal of Laparoendoscopic \& Advanced Surgical Techniques. January/February 2010, 20(1): 77-80. doi:10.1089/lap.2008.0329.

[9] Veena Kumari, Nilay Biswas, Nilanjan Mitra, Hiralal Konar, Dipak Ghosh, and Sukanta K. J.is ligation of hernial sac during orchiopexy mandatory?. Indian Soc Pediatr Surg. 2009

[10] Delikoukos S., Lavant L., Hlias G., et al: the role of hernia sac ligation in postoperative pain in patients with elective tension-free indirect inguinal hernia repair:a prospective randomized study. Hernia. 2007.

[11] Smedberg.. SG, Broome AE, Gullmo A. Ligation of hernial sac? Surg. Clin. North Am. 1984

[12] Gharaibeh KL., Matani YY., To ligate or not to ligate the hernial sac in adults? Saudi med j. 2000 Nov;21(11):1068-70.

[13] Imamuglu M., Cay A., Sarihan H., Ahmetoglu A., Ozdemir O. Paravesical abscess as an unusual late complication of inguinal hernia repair in children. Pediatric Surgery International - volume23, number 4, 349-352

[14] CM, St Peter SD, Bakom A., Murphy PJ, Late abscess formation following indirect hernia repair utilizing silk suture. Pediatric surg. Int. 2007 